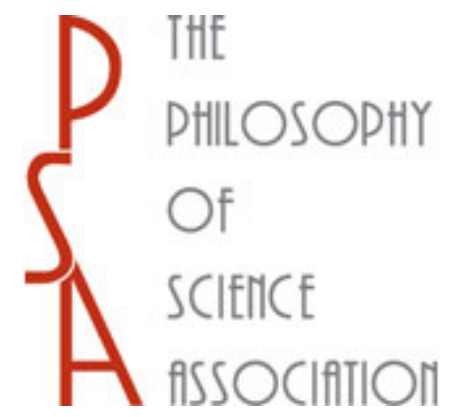

Explanation and Subsumption

Author(s): Robert Cummins

Source: PSA: Proceedings of the Biennial Meeting of the Philosophy of Science Association, Vol. 1978, Volume One: Contributed Papers (1978), pp. 163-175

Published by: The University of Chicago Press on behalf of the Philosophy of Science

Association

Stable URL: http://www.jstor.org/stable/192634

Accessed: 17-09-2015 17:30 UTC

Your use of the JSTOR archive indicates your acceptance of the Terms \& Conditions of Use, available at http://www.jstor.org/page/ info/about/policies/terms.jsp

JSTOR is a not-for-profit service that helps scholars, researchers, and students discover, use, and build upon a wide range of content in a trusted digital archive. We use information technology and tools to increase productivity and facilitate new forms of scholarship. For more information about JSTOR, please contact support@jstor.org.

The University of Chicago Press and Philosophy of Science Association are collaborating with JSTOR to digitize, preserve and extend access to PSA: Proceedings of the Biennial Meeting of the Philosophy of Science Association. 


\title{
Explanation and Subsumption
}

\author{
Robert Cummins 1 \\ University of Wisconsin-Milwaukee
}

\section{The Claim}

Quine once claimed that modal logic derived from a use-mention mistake ([8], p. 196). In this paper, I will peddle an equally outrageous claim: the thesis that subsumption is an essential goal of fundamental explanatory theorizing rests on a failure to distinguish explaining what made $x$ happen from explaining why we believe what we do about what made $x$ happen. I don't mean to suggest that the distinction wasn't noticed (except perhaps by Hume); only that it wasn't vigorously enforced. There have always been philosophers who assimilated a belief to its justification with malice aforethought.

\section{Strategy}

Everyone knows that subsumption is not sufficient for explanation: the length of a pendulum is not explained by appeal to its period and the pendulum law, and emission spectra are not explained by Balmer's formula. But the spirit of the deductive-nomological account of explanation lives on in the belief that subsumption is an essential feature of an explanatory theory. Contextualists have argued with some plausibility that subsumption is seldom necessary to particular explanations, and it has been argued by some (myself in [1]) that subsumption is at best incidental to functional explanation. But even when these points are conceded, the majority opinion, I suspect, is still that non-subsumptive explanation is really a stand-in or under-study: if subsumption under law isn't waiting in the wings somewhere, then the play is a farce. Thus, to attack the claim that subsumption is necessary to explanation, it is not enough to provide an explanation that does not involve subsumption--e.g., the car turned over because the left front tire blew out-for defenders of the subsumptive model have always claimed that such cases are cases of incomplete explanation: promissory notes, but not hard cash. This line was taken by Hempel and Oppenheim [5], and it has some plausibility: when we trot out a "because" but do not or cannot

PSA 1978, Volume 1, pp. 163-175

Copyright (C) 1978 by the Philosophy of Science Association 
trot out a law, we typically suppose nevertheless that there must be a law of the appropriate sort, even if we don't know what it is. It is therefore open to the subsumptivist to claim that it is only the promise of the missing law and a corresponding subsumption that gives the nonsubsumptive explanation point. If we want to block this sort of move, we must set the stage in such a way as to guarantee that there are no unstated laws in the wings. This is what I will try to do: my strategy will be to describe a theory that (1) if true, would explain every event it can describe at all, (2) does not explain by subsumption, (3) is fundamental in that it is understood to be irreducible. If I can do this, I will have shown that the possibility of a thorough-going explanatory theory worthy of the name does not depend for its explanatory import on the possibility of subsumption under law.

\section{ANA}

I call the fanciful theory I am about to describe ANA because the explanatory strategy it exploits is analytical rather than subsumptive. ANA is very much like Lucretian Atomism without the Swerve or the Downward Tendency. According to ANA, the universe is a collection of elementary parts moving relative to one another. These are of several types, distinguished by shape and inertial mass. Momentum and kinetic energy are transmuted and conserved in the usual way. There is no gravitation, charge or anything else of the inverse-square law variety. Collision is king and is perfectly elastic. No elementary part can change its shape or mass.

According to ANA, every event has a unique micro-analysis: just as every thing is a congeries of elementary parts, so every event is a congeries of micro-events. Thus, to explain an event is simply to analyze it into micro-events and explain these. In the simplest case, we explain $0^{\prime} s$ moving from $P_{1}$ to $P_{2}$ by analyzing this event into the corresponding motions of $O^{\prime}$ 's elementary parts, and then explaining those by appeal to the stated properties of elementary parts.

ANA recognizes only one kind of micro-event: instantaneous acceleration of an elementary part. Since shape and mass cannot change, the only candidates are change of position and change of velocity. ANA agrees with classical mechanics in refusing to regard mere change in position as a genuine change of state. Continued motion is the natural state of things: what requires explanation is a change in that state of affairs. The argument here is based on the peripatetic principle that the effect ceases when the cause ceases. Since the only causes are collisions, and since motion continues without continuous impact, it follows that continued motion is not an effect, hence requires no cause to explain it.

According to ANA, then, the only genuine micro-events -- the only real effects -- are accelerations, and these are all caused by collisions. The universe is like a pool table. Thus, the only laws employed by ANA that have a chance of explaining a micro-event are the conservation principles, for they are the only laws that subsume anything that wants 
explaining at the micro-level. One may suspect this is the Achilles' Heel to be exploited by the wily subsumptivist. Perhaps it is. Before we examine ANA's heel, though, let's take a look at the upper body.

\section{Macro-laws}

I'm inclined to believe that ANA might be true of a universe that simply did not exhibit any law-like regularities at the macro-level. If so, then the only candidate for subsumptive explanation would be the one just mentioned. But such a universe would be very different from our own, and so perhaps we should expect that explanation of the events in such a universe would be radically different too. Our concept of explanation was evolved for local employment, so we'd best see if we can allow for macro-laws of a sort without allowing them any explanatory force.

I will begin with a very artificial illustration which I call the alphabet phenomenon. Suppose you are gazing out of your office window and notice that the people on the quadrangle periodically form block letter patterns like a marching band. Further observation yields the result that the letters succeed one another in alphabetical order, cyclically, with 'A' following ' $Z$ '. Initially, you suspect design, but subsequent investigation rules this out. The truth is that the path of each person is simply the shortest path between point of off-quad departure and point of off-quad destination, so that the on-quad position of a given person at a given time is fixed by the position, time and initial velocity of departure, these in turn being determined by similar factors independent of the alphabet phenomenon. Thus, every macro-event subsumed by the Alphabet Law -- i.e., every letter change -has a unique micro-analysis, and each of the analyzing micro-events is independent of the others and independently explicable. Having discovered this, you must conclude that the alphabet phenomenon is just a very striking coincidental artifact of the fact that a lot of people are going their own way. And how striking is it, really? After all, they must exhibit some pattern or other at any given time, and these must succeed one another somehow. What is striking is simply that we happen to have ready to hand, at no charge, a vocabulary for describing it and a corresponding method for predicting it. Generally, adequate taxonomies are not so cheap.

I call things like this artifactual regularities. "Accidental" is misleading: the Alphabet Law supports counterfactuals and 1icenses predictions. It is no accident that the patterns evolve as they do: "B's" following " $A$ " isn't sheer coincidence. What's coincidental is fust that we can describe things in a vocabulary designed for something else. Nor is it law-like in the full sense: knowing what we know, we can see that alphabetical order has no physical significance.

The important point about artifactual regularities is that they have no explanatory force. Consider this: Patterns succeed alphabetically; the last pattern before 2:00 p.m. was A-shaped; therefore, the 2:00 p.m. pattern is B-shaped. As a predictive tool, the Alphabet Law is practi- 
cally indispensible. As an explanatory tool it is a non-starter. This manifests itself in an interesting way: if we ask why the Alphabet Law is true, almost any answer we give will be misleading. Given the microevents that are actually happening, we can see why it will continue to hold. So the question comes to this: why do we find these particular causal chains at the micro-level? This is reminiscent of the Cosmological Argument: granted we can explain each event in the chain, but why this whole chain rather than another? This question has no answer (and doesn't need one), and neither does our question about the Alphabet Law.

Could all macro-regularities be artifactual? Well, why not? One reason we might think not is this. If we think that admitting the artifactuality of a regularity is tantamount to conceding that the events falling under it are inexplicable, we will resist artifactuality. But we have just seen that this worry is unfounded: we recognize that the Alphabet Law is artifactual precisely because we see how the events it subsumes should be explained. (I think, by the way, that some philosophers of language and some cognitive psychologists may have been guilty of this mistake: there are good reasons for insisting on explanations of macro-regularities, but among them is not that the only alternative is the inexplicability of the subsumed events.) There is no doubt that many actual macro-regularities are not artifactual. My point is simply that a good scientific theory, a theory capable of explaining everything needing explanation -- would be possible in a world in which all macro-regularities were artifactual.

Another reason for thinking there must be non-artifactual macro-laws is that there appears to be a simple way to construct them: surely there will be laws like this:

(1) If an event having micro-analysis A-1 were to occur, an event having micro-analysis A-2 would occur.

For just consider a macro-event e occurring at $t$ : it has a micro-analysis $\mathrm{A}(\mathrm{e})$. Now we use our micro-theory - ANA or whatever -- to find the successor at $t^{\prime}$ of each of the micro-events specified by $A(e)$. Taken together, these constitute a macro-event $e^{\prime}$ at $t^{\prime}$, viz., the macroevent whose micro-analysis specifies the successors at $t^{\prime}$ of the events specified by $A(e)$.

This seems okay until we remember that the Alphabet Law is itself of this form: evidently, laws of the form (1) can be artifactual. Indeed, once you think of it, they are bound to be: micro-events don't get together and occur in the way they do in order to constitute a macroevent susceptible of a certain analysis. The succession of events we get by calculating in the manner just rehearsed may well be covered by no macro-law other than those which describe the effect of $E$ as the sum of the micro-effects of the micro-causes constituting $\mathrm{E}$. Macro-laws of that sort are obviously boring: they tell us that the effect of $\mathrm{E}$ is whatever follows next in the causal order, and refers us to the microtheory for details. It might be that this is just the best one could do at the macro-level. 


\section{More on Macro Laws}

In order to tighten our intellectual grip on this possibility, let's consider a non-artifactual macro-regularity, viz., ionic chemical bonding as it is treated in valence theory. Pure valence theory tells us we have a compound - something capable of no further bonding - when the sum of the valence numbers of the elemental constituents is zero. Let's call this the Valence Law. Valence numbers are assigned to elements in such a way as to make this true. At this level, the numbers have no non-dispositional significance: we could multiply them all by 2 or 27 without changing the theory in any important way. What everything having the same valence shares is a certain dispositional property viz., the disposition to bond with anything having the same valence but for sign. Now it might have turned out that the numbers had a straightforward significance at the micro-level which provided an explanation of the bonds subsumed by the theory. For instance, it might have happened that molecules with a valence of $t n$ had $n$ free hooks, and molecules with a valence of $-n$ had $n$ free eyes. This would provide a straight-forward explanation of why the Valence Law holds true. In particular, it would explain why things having the dispositional property specified by a given valence number have that disposition.

Given this unified account, explanation of bonding by appeal to valence is surely unproblematic: the Valence Law, together with specifications of the relevant numbers, explains why we get $\mathrm{NaCl}, \mathrm{H}_{2} \mathrm{O}$, and so on. Actually, of course, there are no hooks and eyes in the picture. But rather than consider the actual case, imagine a rather different fantasy: suppose there is no single explanation of ionic bonding, but several. Sometimes it is hooks and eyes, sometimes something else. So the things that have a disposition to form ionic bonds have it for different reasons. To elaborate the Lucretian fantasy, perhaps some atoms are equipped with one or more clamps, like lobster claws: what can be grabbed depends on the size, shape and number of claws, together with the size and shape of the things to be grabbed.

How does explanation by subsumption under the Valence Law fare under this revision?

Not so well, I think. For now subsumption under the Valence Law is subsumption under a statement that generalizes across explanatory boundaries. Consider this:

(i) sodium bonds 1-1 with chlorine because (ii) sodium has a valence of +1 and (iii) everything having a valence of +1 bonds 1-1 with chlorine.

(iii) remains true, of course, but it's a put-up job: the uniformity it presents us with is artifactual. This becomes obvious as we increase the heterogeneity of the physical situations underlying (iii): what if there are as many different micro-stories as there are elements? (There aren't, but this is surely a contingent matter.) (iii) would lose all semblance of explanatory force: for it would obviously be nothing more 
than a convenient recursive way of generating a list of facts like (i). I say "nothing more" because the facts listed would not hold in virtue of anything corresponding to our method of generating the 1ist. Under these conditions the Valence Law would be exactly comparable to the Alphabet Law.

Another way of putting the point is this: (iii) attributes a disposition - the disposition to bond 1-1 with chlorine - to a specific list of elements. But the physical stories underlying this disposition are as numerous as the items on the list. So what (iii) tells us is that sodium is disposed to bond with chlorine for some reason or other which is, in fact, peculiar to the case of sodium. So the "explanation" comes to this: sodium is disposed to bond 1-1 with chlorine for some special reason. Moliere would be pleased.

I want to draw two morals from this story: the first is that the explanatory power of the Valence Law depends on what we know or believe about the facts underlying particular bonds. In the absence of such knowledge or belief, its explanatory force should be zero, for we cannot actually explain anything to ourselves by appeal to a regularity we do not actually know or believe to be non-artifactual. The second is that it is a purely contingent matter that macro-regularities such as the Valence Law are non-artifactual and hence explanatory. This is to be strongly distinguished from the claim that it is purely contingent that ionic bonds can be explained: in the fantasy just rehearsed, we became convinced that the Valence Law is artifactual precisely because of the way we do explain ionic bonds.

\section{Micro-laws}

If the foregoing is correct, we must admit the possibility of a scientific theory capable of explaining every macro-event without subsuming any since micro-analysis could reveal that all macro-laws are artifactual. If there is to be any subsumptive explanation in the picture, it must be explanation of micro-events. ANA admits of only one sort of micro-event -- accelerations -- and only one sort of explanation of them -- collisions. So our question reduces to the question whether billiard-table physics is explanation by subsumption. We can further simplify matters by supposing that every elementary particle has the same mass -- i.e., that the conservation principles (CV) reduce to conservation of $\mathrm{v}$ and $\mathrm{v}^{2}$.

Is subsumption under CV explanatory? I want to approach this matter via a different but related question: when a particle conforms to $\mathrm{CV}$, does it manifest a disposition? On the face of it, to say the elementary particles satisfy $\mathrm{CV}$ is to attribute to them a disposition: $\mathrm{CV}$ tells us what a particle would do if ... without telling us why. Furthermore, this disposition is a "brute disposition" there being (given our assumptions) no theoretical prospect of explaining why particles satisfy CV. Brute dispositions are unwelcome for this very reason, a reason made famous (though not clear) by Moliere. Asked why opium puts people to sleep, Moliere's doctor replies that opium has a dormitival 
virtue. The prospect of having to take seriously at the level of elementary particles what we regard as a joke at any other level is sufficlently unwelcome to make us ask whether we are really dealing with a genuine disposition here. One naturally tends to think of conserving velocity, for instance, as the manifestation of a disposition, viz., the disposition to follow the roads and speed limits allowed by the parallelogram rule. But there are indications that this is a mistake.

FIRST: If conserving velocity and its square -- moving we11, as I shall say -- is the manifestation of a disposition, it is peculiar in that the disposition is one that everything has (according to ANA). Thus there would be no point in asking why things have this disposition in the sense in which this means: how do the things that have it differ from the things that do not? This contrasts sharply with uncontroversial dispositions such as flexibility where the need for explanation derives in part from the fact that not everything is subject to the law of flexible things. We want to know about just these things what accounts for the fact that they would flex were they stressed whereas other things would not. No comparable question can be ralsed concerning the disposition to move well. SECOND: if moving well is a manifestation of a disposition, then it is peculiar in that the disposition is always manifested, never latent. Again, this contrasts with uncontroversial dispositions. Soluble things are sometimes dissolving, sometimes not. This is the point of the familiar subjunctive formulation: we use the conditional to represent the connection between immersions and dissolvings, and the subjunctive because there may be no immersions. A thing may be soluble but not dissolving because conditions are not propitious. The need for explanation derives here from the need to show why the propitious conditions are propitious, i.e., from the need to explain how manifestations are brought about. Once we know this, we can explain, e.g., why sugar dissolves when it does, and why it doesn't when it doesn't. Since the distinction between having the disposition and manifesting it is absent in the case of moving well, the corresponding need for explanation is absent as well. Conservation is not a sometimes thing: there are no special conditions under which things move well, conditions that are sometimes present, sometimes not: hence there is no pressure to explain why some conditions are propitious for moving well while others are not. This is reflected in the fact that a subjunctive formulation is inappropriate. The subjunctive formulation allows for the possibility that the conditions requisite for manifestation may not exist. But there is nothing comparable to allow for in the case of moving well: a particle would move well were it to be the case that ... what? Anything whatever.

Dispositions call for explanation because they are not universal and because they are not always manifested. Brute dispositions cause problems because the required explanations are not and cannot be forthcoming. But $\mathrm{CV}$ raises no questions comparable to those raised by flexibility or solubility, and hence we need not be embarrassed for want of comparable answers. It is a mistake, therefore, to think of moving well as the manifestation of a disposition, brute or otherwise. This is not yet to say that it makes no sense at all to ask why particles move-we11: it is 
only to say that asking this is not like asking why soluble things dissolve. This may seem small comfort. If the only possible answer to "Why do particles move well?" is "They just do." doesn't the spirit of Moliere's criticism remain untouched?

I think the way is prepared for a defense of the short answer to the question about moving well, and hence for a negative answer to our question about Moliere's criticism, once we are clear that in giving the short answer we are not acquiescing in a brute disposition. If we think "Why do particles move well?" is analogous to, "Why do soluble things dissolve?" we will think of ourselves as asking after the causes of moving well. For in order to explain why soluble things dissolve we must uncover the etiology of dissolution. Once we construe the question about moving well in this way, the short answer is bound to seem indefensible. We have seen ample reason to resist this construal, however, and this leads me to wonder whether the short answer is defensible after all.

The best way to approach this matter, I think, is by examining another case in which the short answer is unproblematic. Suppose someone were to ask why things move or have sizes or shapes. We might reply that, while it makes perfectly good sense to ask why this or that particular thing has the motion (shape, size) it does, it does not make sense, or anyway not the same sort of sense, to ask why anything at all moves (has a shape or size). This is just the way things are. The question would make sense were we to suppose that there was a time when nothing moved (had a shape or size), for then we might be asking what brought about the onset of motion (shape, size), i.e., what brought about the change in the universe from its supposed previous state to its present one. But, of course, we don't suppose any such thing. Things do change their motion (size, shape), and hence it makes sense to ask how some particular thing came to have the motion (size, shape) it does now have. But there is no comparable question concerning things generally, for the universe didn't change from a motionless state.

This is the stance taken towards inertial motion in classical mechanics: mere change in position is not regarded as a change of mechanical state, and hence it does not require mechanical explanation. Now it seems to me that this stance can and should be taken towards moving well. We may ask why this or that thing is moving well in the way it is, but we cannot in the same way ask why things move well rather than not. Once we cease to see moving well as the manifestation of a disposition, there is no need to see it as involving a change in mechanical state at all, hence no longer any reason to suppose that moving well must be explained. There is, in other words, no reason to suppose that "Why do things move well?" cannot be classed with "Why is there motion (shape, size)?" and given the short answer (as I did in [2]).

If there is no reason why things move well -- why they satisfy CV -then we cannot, it seems to me, explain a particular case of moving well by appeal to CV. If there's no reason why particles move well, there's no reason why this particle moved well then. A particle doesn't move 
well because velocity and its square are conserved: its moving well is velocity and its square being conserved.

If $\mathrm{CV}$ does not explain why a particular particle moved well on a particular occasion, perhaps it explains why that particle took the particular direction and speed it took. Or is it the initial velocities and positions that explain this? Asking the question in this way is an open invitation to stipulation, or analysis of ordinary language. We'd best find another way of raising the question.

Let's consider a case in which A and B collide "head-on", B initially at rest (relative to the observer), A moving at $2 \mathrm{~m} / \mathrm{sec}$. What happens is that A stops and $B$ assumes $A$ 's initial velocity. The event to be explained here is B's assuming a velocity of $2 \mathrm{~m} / \mathrm{sec}$, or rather this particular change in B's velocity, viz., from zero to two. Let's call this event e. Rather than ask why e occurred, let's ask: (I) what made e happen? This may or may not be a different question, but it's easier, and that's reason enough to shift to it.

Now certainly the laws didn't make it happen. ('Principle' used to (maybe still does) have an ambiguous use in this connection: When a 17 th Century writer referred to the principles governing something's behavior, this was ambiguous as between something that makes the behavfor happen and a statement subsuming that behavior.) What made it happen was the collision with $\mathrm{A}$, this being, in fact, a collision headon with something traveling at $2 \mathrm{~m} / \mathrm{sec}$ due north. Let's call the collision c. The answer to our question then is: $c$ made e happen, and this answer involves no appeal to laws at all. Of course we may have to appeal to laws to justify our claim that it is $c$ that made $e$ happen, but that is plainly another matter altogether. We mustn't confuse what made e happen with what justifies us in believing $c$ made e happen.

Is there anything for subsumption to do in connection with answering (I) besides form part of a justification of our answer? I think not. We might go on to ask: Why or how did c make e happen, as opposed to $\mathrm{e}^{\prime}$, say? But this question has no answer at all: there isn't any way in which $c$ made e happen: it just did. Since this question has no answer, subsuming $e$ under $C V$ can have no role in answering it.

What do we do when we subsume an event, or rather a pair of events, under a law? Well, in our illustrative case, CV gives us:

$$
\begin{aligned}
& \text { (1) } 4=V_{A}^{2}+v_{B}^{2} \\
& \text { (2) } 2=V_{A}+v_{B}
\end{aligned}
$$

where $V_{A}$ and $V_{B}$ are the final velocities of $A$ and $B$. Elementary algebra reveals that either $V_{A}=2$ and $V_{B}=0$, or $V_{A}=0$ and $V_{B}=2$. Since $A$ cannot pass through $B$, we have:

(3) $\mathrm{V}_{\mathrm{A}}=0$ 
(4) $\quad V_{B}=2$

The theory and initial values give us a certain mathematical representation of the situation, viz., (1) and (2). A little algebra allows us to substitute (3) and (4) for (1) and (2). What the algebra does is allow us to make it obvious that the required representation is in fact the representation of a situation in which A stops and B continues at a velocity of $2 \mathrm{~m} / \mathrm{sec}$. So subsumption of $(c, e)$ under $\mathrm{CV}$ comes to this: we construct a mathematical representation by plugging initial values into $\mathrm{CV}$. We then do enough algebra to make it obvious that the representation we have constructed is a representation of $e$.

Is this "explaining why e happened?". Maybe. But it isn't explaining what made e happen, for it presupposed the answer to that question: we start by characterizing $c$, the event we suppose made e happen. In the absence of any supposition about what made e happen, subsumption cannot get off the ground.

Let's consolidate:

(1) Subsuming (c, e) under $\mathrm{CV}$ is irrelevant to answering (I): "What made e happen?"

(2) The answer to (I) is a singular causal statement.

(3) Justifying singular causal statements often involves appeal to subsuming laws.

(4) Subsumption presupposes an answer to (I), since we begin by describing the event that made $e$ happen.

If subsuming (c, e) under CV explains why e occurred, then explaining why e occurred is different from answering (I). Or rather, since answering (I) is patently part of explaining why e occurred, the contribution of subsumption has to lie in answering whatever question is left over when we take away (I). To borrow an old trick of Wittgenstein's, we want to know what remains when we subtract explaining what made $e$ happen from explaining why e happened. I think the remainder is a justification of the claim that $c$ made $e$ happen.

The right way to view matters, I think, is this: when we explain why e happened we do two very different sorts of things:

(1) we say what made e happen, viz., c.

(ii) we justify the claim that $c$ made e happen by subsuming $(c, e)$ under $C V .^{2}$

How does subsuming (c, e) under CV justify (i)? Well, c certainly occurred. And when we describe c-- write out the equations that depict its mechanical features, -- we find we have depicted e.

Thought of in this way, the CV principles are depiction rules -rules or schemata for constructing representations of mechanical events, representations which are high in epistemological utility. This sounds bizarre, not to mention instrumentalist, until we recall two points: 
(1) there is nothing instrumentalist about saying $c$ made $e$ happen;

(2) causal claims are justified - often enough - by appeal to laws.

What my claim boils down to is this: to explain an event via ANA is to cite its cause, and to justify this citation.

\section{Taking It Back}

Now, haven't I given up the claim that subsumption is irrelevant to explanation? Yes and no. "Yes" because the complex business we call explanation typically requires specification of causes, these need to be justified, and that's typically achieved via subsumption. "No" because subsumption is given a subsidiary justificatory role which might be played by other actors. Suppose we didn't need to justify singular causal statements by appeal to laws, i.e., suppose we could just tell, without appeal to "constant conjunction" or anything similar, that $c$ caused e. Davidson([3], [4]) has argued that singular causal statements do not entail causal laws. This shows that we can understand a singular causal statement without knowing any corresponding law. And once we abandon the Humean claim that causal laws are involved in the analysis of singular causal statements, the doctrine that they are necessarily required for the justification of singular causal statements loses its plausibility. It's rather obvious on the face of it that we often make justified singular causal statements without any serious idea of how to generalize them.

\section{Diagnosis}

What cannot be accomplished in the absence of laws is serious, quantitatively accurate, prediction, and this, I cannot help but believe, is what's behind the subsumptivist account of explanation. It all goes back to Hume's conflation of two problems:

(i) the analysis and justification of singular causal statements; (ii) the analysis and justification of predictive inferences.

Whatever Hume intended to treat, what he managed to treat was mainly (ii). A review of illustrations of "causal reasoning" in the Treatise [7], and Inquiry [6] reveals few cases clearly involving singluar causal judgments. And the classic argument -- that you cannot infer the effect from a knowledge of the cause, is a spectacular howler if directed at (i): surely to make a relational judgment requires knowledge of both relata!! (An analogous argument will show that singular size comparisons cannot be justified!!)

Hume has a doctrine about (i): viz., that singular causal statements entail and must be justified by appeal to subsuming laws, but this doctrine is obviously false. It only seemed plausible when (i) and (ii) were not distinguished.

This will seem high-handed to many: there is a long and venerable tradition in philosophy which assimilates truth conditions to evidence 
conditions on purpose. Philosophers who do this will be unimpressed by a doctrine that depends on distinguishing what makes a singular causal statement true from what justifies it. It is not within my intention nor my ability to take on this tradition. My intention is rather to indicate why subsumptivist accounts of explanation are not likely to find a secure home outside that tradition. My argument took the form of a categorical rejection of subsumptivism only because I cannot find a secure home within that tradition myself.

\section{Notes}

${ }^{1}$ I should like to thank John Koethe, Warren Ingber, David Zaret, and Paul Teller for their stimulating attempts to talk me out of most of what follows. I was similarly aided by stimulating audiences at Chicago Circle and The University of Wisconsin where I presented an earlier draft of this paper.

${ }^{2}$ Compare Michael Scriven's views in [9]. I'm inclined to think that one of the things that makes "really" explanatory theories seem so interesting and important is that they provide justifications that "make us understand" an event's occurrence. Hence, we feel cheated when provided a purely inductive or authoritative justification in an explanatory context. 
$\underline{\text { References }}$

[1] Cummins, Robert. "Functional Analysis." The Journal of Philosophy 72(1975): 741-765.

[2] --_-_-_tates, Causes and the Law of Inertia." Philosophical Studies 29(1976): 21-36.

[3] Davidson, Dona1d. "Actions, Reasons, and Causes." The Journal of Philosophy 60(1963): 685-700.

[4] -Causal Relations." The Journal of Philosophy 64(1967): 691-703.

[5] Hempel, C. and P. Oppenheim. "Studies in the Logic of Explanation." Philosophy of Science 15(1948): 135-175.

[6] Hume, David. An Inquiry Concerning Human Understanding. ed. Charles W. Hendel1. Indianapolis: Bobbs-Merrill, 1955. (Originally published 1748 as Philosophical Essays Concerning Human Nature. Reprint of 1777 edition.)

[7] --- A Treatise of Human Nature. ed. L.A. Selby-Bigge. Oxford: Oxford University Press, 1951. (Books I and II originally published 1739 and Book III 1740. Reprint of 1888 edition.)

[8] Quine, W.V. Word and Object. Cambridge, MA: MIT Press, 1960.

[9] Scriven, Michael. "Causation as Explanation." Nous 9(1975): 3-16. 